\title{
Validez y confiabilidad de la Escala de Resiliencia (SV-RES) para adultos mayores en Lima metropolitana
}

\section{Validity and reliability of the Resilience Scale (SV-RES) for older adults in metropolitan Lima}

Sheyla Janine Moscoso-Escalante

$\triangle$ sheyla.moscoso.e@gmail.com

Universidad Peruana de Ciencias Aplicadas, Lima, Perú.

Ana María Castañeda-Chang (i)

$\triangle$ pcpsacas@upc.edu.pe

Recibido: $31-08-2018$

Universidad Peruana de Ciencias Aplicadas, Lima, Perú.

Aceptado: 07-11-2018

Publicado: 17-12-2018

\section{Resumen}

El objetivo principal del presente estudio fue analizar la validez de constructo y la fiabilidad de la Escala de Resiliencia (SV-RES) de Saavedra (2008) en adultos mayores debido a las escazas investigaciones en torno a la variable, la cual funciona como factor protector para el envejecimiento saludable. Es un muestreo no probabilístico conformado por trescientos adultos mayores pertenecientes a un centro del adulto mayor de Lima Metropolitana, entre los cuales el $43 \%$ estuvo conformado por varones y el $57 \%$ por mujeres entre sesenta y 96 años. Se aplicó el SV-RES de Saavedra (2008) y el RESIM, adaptado por Palomar y Gómez (2010) para el análisis convergente. Como resultados, el análisis factorial exploratorio del SV-RES arrojó una solución de tres factores (yo soyestoy, yo tengo, yo puedo) que explicaron el 50,60\% de la varianza total. El análisis factorial confirmatorio ofrece resultados adecuados: $X^{2}=108.779,922$, $\mathrm{RMSEA}=$ 0,028 , y CFI $=0,996$. Las correlaciones entre la escala de resiliencia SV-RES y RESI-M fueron positivas y significativas. Mediante el uso del coeficiente alpha de Cronbach se obtuvieron valores mayores a 0,70. Estos resultados permiten concluir que el SV-RES y sus puntuaciones son una medida válida y fiable para medir la resiliencia en adultos mayores.

Palabras clave: Adulto mayor, resiliencia, validez, confiabilidad, análisis factorial exploratorio. 


\section{Abstract}

The main objective of the present study was to analyze the construct validity and reliability of the Resilience Scale (SV-RES) of Saavedra (2008) in older adults due to the lack of research around the variable which works as a protective factor for healthy aging. It is a non-probabilistic sampling consisting of 300 older adults belonging to a center of the older adult of Metropolitan Lima of which $43 \%$ were male and $57 \%$ female, between 60 and 96 years. For the analysis, were applied the instruments of SVRES by Saavedra (2008) and RESIM by Palomar y Gómez (2010). For the results, the exploratory factor analysis of the SV-RES showed a three-factor solution (I am, I have, I can) that explained $50.60 \%$ of the total variance. The confirmatory factor analysis offers adequate results: $X^{2}=108,779.922$, RMSEA $=0.028$, y CFI $=0.996$. The correlations between the resilience scale SV-RES and RESI-M were positive and significant. Using the Cronbach's alpha coefficient, values greater than 0.70 . These results allow us to conclude that the SV-RES and its scores are a valid and reliable measure to measure resilience in older adults.

Keywords: Older adult, Resilience, Validity, Reliability, Exploratory Factor Analysis.

\section{Introducción}

En diferentes investigaciones se destaca la importancia y relevancia de la resiliencia centrada en la capacidad de los niños para adaptarse y sobrepasar las adversidades de su entorno. Como diría Becoña (2006), el origen del estudio de la resiliencia es conocer, en psicología, la etiología y desarrollo de la psicopatología, especialmente en niños que están en riesgo a desarrollar patologías debido al sistema familiar y entorno hostil. Por tanto, al seguir la línea de búsqueda de estudios e investigaciones, se encuentra que unos de los campos en donde más se centra o tiende a centrarse la resiliencia es en el de los trabajos con niños y adolescentes en situaciones de riesgo como abandono, pobreza extrema y embarazo adolescente, entre otros (Alcalde, Alcalde y Palacios, 2011; Benvenuto, 2015; Retiz, 2016). Surgen, de esta manera, diversos constructos para medir la resiliencia, como ocurre en los trabajos de Salgado (2005) o Connor y Davidson (2003).

Si bien es importante la promoción y prevención de la resiliencia en los jóvenes, existen grupos etarios en los que las investigaciones y estudios son escasos, como es el caso del adulto mayor, grupo en el que es importante detenerse aún más y reforzar las investigaciones, ya que la resiliencia es vista como un factor protector para el envejecimiento saludable (Resnick, 2014). Asimismo, se propone la resiliencia como un componente para la adecuada adaptación psicosocial, en consecuencia, es importante que el adulto mayor la considere como una herramienta para el mejoramiento de su calidad de vida (Cortés, Flores, Gómez y Reyes, 2012).

Es importante conocer que el periodo de vida del adulto mayor es caracterizado por factores de protección que fortalecen la resiliencia, tal que suelen afrontarse eventos críticos como son los problemas de salud, la pérdida de seres queridos y, en muchos casos, un decremento en su capacidad económica (Fernández, 2004; Gómez, 2010; Jiménez, 
2011). A pesar de esto, hay estudios que indican que, aunque se hayan sufrido tales dificultades y muchas veces circunstancias extremas, peligrosas o traumáticas (guerras y austeridad), muchas personas mayores se encuentran en adecuada condición de salud, sin tomar en cuenta los padecimientos propios de la edad, e incluso dan muestras de vitalidad, buen humor y entusiasmo, si realizan actividades que los mantengan ocupados y que aporten beneficios para ellos y para otras personas; además, poseen gran interés en seguir contribuyendo y participando con su familia y amigos (Jiménez, 2008); proceso este que se vuelve significativo y apreciable en los adultos mayores (Severina, 2017).

En cuanto a las investigaciones en nuestro contexto se pudo encontrar un estudio realizado en Trujillo en donde se busca brindar evidencias psicométricas del instrumento Brief-Resilient Coping Scale (BRCS) en adultos mayores peruanos (Caycho, Ventura, Garcia y otros, 2018). Esta medida unidimensional breve de Resiliencia cuenta con 4 items que evalúa la capacidad de los individuos para hacer frente al estrés de manera adaptativa; en contraste, la escala de resiliencia (SV-RES), elaborada por Saavedra y Villalta (2008b), permite la identificación del grado de resiliencia consigo mismo, frente situaciones adversas y también describe la interacción del sujeto con su entorno y con sus recursos, brindándose un perfil más amplio del individuo.

En tal sentido, la escala de resiliencia (SV-RES), elaborada por Saavedra y Villalta (2008b), permite la identificación del grado de resiliencia como una interacción consigo mismo, con las demás personas y sus recursos en relación a diferentes situaciones adversas para poder aprender y transformarlas de acuerdo a los niveles de estructuración de conciencia del individuo enfocado en sus condiciones base, visión de sí mismo, visión del problema y respuesta resiliente

Esto se debe a que su base teórica está basada en el modelo de Grotberg (1995), que refiere que la resiliencia no es un factor estático, sino que va cambiando con la edad debido a las diferentes etapas del ciclo vital del ser humano, ya que las adversidades que se pueden ir presentando son distintas. Por lo tanto, se entiende que es un proceso continuo y se puede decir que la resiliencia nunca es absoluta ni decisivamente estable (Munist, 1998 citado en Benvenuto, 2015).

Por lo tanto, ambos modelos, tanto el de Grotberg como el de Saavedra, abordan la realidad del sujeto que contribuye a la constitución de la resiliencia y las modalidades interactivas de su manifestación en el habla. Es decir que cuando el sujeto habla de sí, expresa una interpretación de sí, de los otros y lo que posee para incorporar y abordar las situaciones su vida (Grotberg 1995 y Saavedra y Villalta, 2008b).

Es así como se obtienen doce factores de resiliencia que describen la interacción del sujeto consigo mismo, los otros y sus posibilidades, lo que nos brinda un perfil del sujeto. Estas serían las que responden al "yo soy/yo estoy":

a) La "identidad" se refiere a los juicios generales a la que opta el individuo para interpretar hechos y acciones de modo estable a lo largo de su vida, que lo define y constituye como parte de su caracterización personal.

b) La "autonomía" comprende al vínculo que el sujeto establece consigo mismo para definir su aporte al entorno sociocultural. 
c) La "satisfacción" describe los juicios que tiene la forma particular en una situación problemática.

d) "Pragmatismo" indica el sentido práctico para evaluar y enfrentar los problemas y orientar las acciones que realiza.

Mientras tanto, las que responden al "yo tengo" son:

a) "Vínculos" se refiere al valor de la socialización y redes sociales con raíces en la historia personal como relaciones vinculares, apego, sistema de creencias, entre otros, que sirven de base para la formación de la personalidad.

b) "Redes" se define como los vínculos afecticos que establece la persona con su entorno social cercano y constituyen un apoyo para el sujeto.

c) "Modelos" hace referencia a que el sujete busca en sus redes sociales cercanas una guía para enfrentar sus problemas y superarlos. También se toman en cuenta las experiencias anteriores que sirven de referente frente a la resolución de distintas problemáticas.

d) "Metas" son los juicios que refieren al valor contextual de metas y objetivos definidos, dirigidos hacia un fin.

En cuanto al "yo puedo" se encuentran:

a) La "afectividad" es el auto-reconocimiento de la vida emocional del sujeto y se basa en las posibilidades sobre sí mismo y el vínculo con el entorno (tono emocional, humor, empatía).

b) La "autoeficacia" define las posibilidades de éxito que la persona reconoce en sí mismo como los límites, control de impulsos, responsabilidad ante los actos y manejo de estrés ante una situación problemática.

c) El "aprendizaje" indica poder rescatar la posibilidad de aprender y el crecimiento frente a una situación problemática. De esta manera, se aprovecha la experiencia vivida, se aprende de los errores y se evalúa el actuar.

d) La "generatividad" se refiere a la posibilidad de pedir ayuda a otros, crear y construir respuestas alternativas y planificar las acciones.

De esta manera, con los factores escritos anteriormente es como se puede establecer, definir y distinguir un perfil de resiliencia basado en la interacción consigo mismo, con las demás personas y sus recursos en relación a diferentes situaciones adversas para poder aprender y transformarlas de acuerdo a los niveles de estructuración de conciencia del individuo enfocado en sus condiciones base, visión de sí mismo, visión del problema y respuesta resiliente (Saavedra 2003).

A partir de la investigación piloto del constructo para la validación estadística del cuestionario, se obtuvo un alpha de Cronbach de 0,92. Asimismo, se realizó un estudio para medir el nivel de resiliencia en un grupo de jóvenes sin discapacidad y con discapacidad motora. Un estudio comparativo midió el nivel de resiliencia en un grupo de jóvenes y obtuvo diferencias significativas en ambos grupos. También realizaron un estudio comparativo en personas entre quince y 65 años, para comparar los niveles de resiliencia entre los distintos tramos de edad, y no encontraron diferencias significativas entre el sexo y la edad (Saavedra y Villalta, 2008a). Sin embargo, aún no se ha realizado 
un análisis factorial de la prueba. Por lo que la presente investigación busca analizar las propiedades psicométricas del SV-RES en un grupo de adultos mayores en Lima metropolitana para su adaptación al entorno peruano.

Cabe señalar que la distribución por edades de la población mundial está pasando por una profunda transformación, cada vez existe más población adulta en nuestro entorno y esta vive por años. Sobre este aspecto, Benavides (2012) menciona que a medida que las tasas de mortalidad y fertilidad han ido descendiendo, la distribución por edades se ha modificado gradualmente, lo que ha impulsado esta transición demográfica, de manera que se ha aumentado no solamente la esperanza de vida, sino la representatividad de población vieja dentro de la población general y, de esta manera, a futuro aumentará paulatinamente (Cárdenas y López, 2010).

Benavides (2015) señala que la esperanza de vida ha aumentado drásticamente, de 46 a 68 años y se espera que llegue a los 81 para finales del siglo, por otro lado, la ONU también estima que para 2050 más del $20 \%$ de la población mundial estará conformada por adultos mayores (Naciones Unidas, 2011). Por lo tanto es sumamente necesario contar con políticas que presten atención a esta edad y logren impactar de manera positiva en sus vidas, debido a los problemas que enfrentan.

En el contexto de nuestro país, según el Instituto Nacional de Estadística e Informática (INEI, 2015), el 9,7\% de la población total está conformada por adultos mayores y la esperanza de vida subió a 74,6 en promedio, con miras a alcanzar los 79 años para 2050. Estos cambios demográficos demuestran que, en nuestra población, las personas también son cada vez más longevas. Es por esto que es sumamente necesario contar con políticas que presten atención a esta edad y logren impactar de manera positiva en sus vidas ofreciendo calidad de vida y un envejecimiento exitoso.

Esta investigación se centra en la validación del instrumento de resiliencia en adultos mayores que se basen en la realidad peruana y no en otras culturas. El conocer los aportes de dicha variable, que toma en consideración, factores propios de una edad adulta, caracterizada por duelos o pérdidas, jubilación, cambios físicos y emocionales permitirá desarrollar programas de prevención y promoción mediante los aportes de las investigación futuras.

Resiliencia en adultos mayores. La mayor parte de los estudios e investigaciones publicadas se centran en una población de niños y adolescentes y, algunas, en la edad adulta. Por lo tanto, se puede ver que, en el grupo etario conformado por adultos mayores, las investigaciones son escasas, y en nuestra sociedad en particular son nulas. A partir de esto, Quintanilla (2000, citado en Quiroz, 2014) plantea al envejecimiento como un proceso normal de cambios, relacionados con el paso del tiempo, que se inicia al nacer, que continúa a lo largo de la vida, y dentro del cual la ancianidad es su fase final.

Sobre estos aspectos, Manciaux (2005) señala que la diversidad de las actividades, romper la rutina, cultivar y hacer amigos, desarrollar la vida espiritual, permanecer activo, pero también prepararse y estar preparado para meditar sobre los acontecimientos o sucesos propios de la vida, nos muestra, de algún modo, el aprendizaje de la resiliencia y la incorporación de la misma. De esta manera, se puede hablar en todo su esplendor sobre un proceso progresivo que se va integrando mediante el pasar de los años y 
la experiencia de haber cometido errores, levantarse, reponerse a distintos duelos y enfermedades, y seguir de pie, demostrando así, de distintas maneras, los diversos caminos de la resiliencia.

En un estudio realizado por Palacios (1994), en el que exponen temas de resiliencia y envejecimiento exitoso, se observa que los adultos mayores mantienen una práctica espiritual; esto se convierte en una fortaleza que les ayuda a reducir la incertidumbre y los temores, así como a sostenerse en las situaciones difíciles y a superar las crisis. En el área psicológica se destacan los recuerdos, la fortaleza individual, el humor, el sentirse útiles. Entre los recursos sociales se encontró que los más sobresalientes son la capacidad de comunicarse, las relaciones frecuentes con familiares y amigos, la recreación, las redes de apoyo y la pertenencia. El aprendizaje acumulado en las diferentes etapas les permitió adquirir la capacidad para adaptarse positivamente a los cambios y situaciones para así superar las adversidades.

Por lo tanto, desde la perspectiva del ciclo de vida, se ha planteado que en edad adulta y en los adultos mayores, la resiliencia, a pesar de los eventos desfavorables, de los retos y pérdidas, es un punto importante para poder tener logros deseados en esta etapa, ya que se invita al sujeto a recurrir a todos los recursos y fortalezas adquiridas a lo largo de la vida, para ser puestos en práctica, así como otras estrategias en compensación a los cambios fisiológicos que el cuerpo puede pasar. Estas recompensas van ligadas al goce del vínculo familiar, buen humor, mantenerse proactivos y preocuparse más por su salud (Jiménez, 2011; Gómez, 2010).

Según Wells (2010), la posesión de altos niveles de resiliencia puede ser un factor que ayuda a los adultos mayores a adaptarse a las dificultades asociadas con el envejecimiento, asimismo identificó que en las redes familiares más fuertes, con los ingresos familiares más bajos y un buen estado de salud mental y física, se encontró una relación significativa con una alta capacidad de resiliencia; por el contrario, la ubicación en la que residen los adultos mayores no afectó esta capacidad, pero lazos sociales fuertes y buena salud mental y física se asociaron con la capacidad de recuperación.

Por lo mencionado anteriormente, se indicaría que más allá del alcance económico de cada persona, lo que lleva a un bienestar psicológico y emocional son los vínculos saludables establecidos en su entorno, los cuales generarán una buena capacidad de adaptación y bienestar a pesar de la adversidad. En consonancia con esta idea, en una investigación de Quiceno y Vinaccia (2011), sobre la resiliencia desde la perspectiva de una enfermedad crónica en población adulta, se aprecia que el apoyo social, las creencias espirituales, la auto trascendencia, optimismo, autoestima, autoeficacia, autocontrol, sentido y propósito en la vida, y el afecto positivo son variables que favorecen y modulan la resiliencia y sirven como factores de protección hacia la salud, por lo tanto, se puede inferir que esos aspectos son los que apoyan y reafirman una capacidad resiliente en esa edad.

Jaque y Mardones (2009) mencionan que el estudio realizado por Barrón (2006) en Chile, encontró que la gran mayoría de adultos mayores no tiene una actividad laboral, por lo que se presenta de esa manera una desventaja para el proceso del envejecimiento, lo cual indica que el trabajo productivo después de los 65 años promueve un envejecimiento sano y esto fortalece una actitud resiliente en los adultos mayores. 
Además, en países en desarrollo existe una deficiencia en los salarios y sistemas de salud para este segmento poblacional (Índice Global del Envejecimiento, IGE, 2014, citado en Benavides, 2015). Esto no se encuentra lejano a nuestra sociedad, ya que el adulto mayor a cierta edad ya no es considerado como un sujeto productivo o como agente aportador en la sociedad, por el contrario, se le considera una carga. A su vez, existe un déficit de programas de salud para los adultos mayores. Sobre este punto, Orosco (2015), en su estudio sobre la depresión y autoestima en adultos mayores institucionalizados y no institucionalizados, señala la necesidad de demostrar que la depresión y baja autoestima no son el resultado de un desencadenante natural del envejecimiento, sino que resultan de la percepción negativa que la sociedad le asigna a este grupo. Según el autor, se deben realizar investigaciones sobre temas que afectan a los adultos mayores, tales como resiliencia, para poder generar aportes teóricos para la concepción de intervenciones y estrategias preventivas y así lograr mejorar o mantener una buena calidad de vida en este grupo generacional, mediante la promoción del estudio de sus problemas dentro de una realidad peruana. Este punto va de la mano con la importancia de la investigación y desarrollo de la escala de resiliencia para adultos mayores.

Objetivo del estudio. Estudiar las propiedades psicométricas de la escala SV-RES en un grupo de adultos mayores de sesenta años a más en la ciudad de Lima metropolitana.

\section{Método}

Participantes. Para la obtención de la muestra se empleó un muestreo no probabilístico de tipo intencional; de este se obtuvo un total de trescientos adultos mayores inscritos en los centros de adulto mayor de un distrito de Lima. Dentro de los criterios considerados para la participación en el estudio, los sujetos deben tener como mínimo de sesenta años, ser autónomos para las actividades de la vida cotidiana y no tener deterioro cognitivo. Las edades estuvieron comprendidas entre los sesenta y 96 años $(M=75,59$, $D E=8,30)$. La distribución del sexo fue de $43 \%$ en varones y el $57 \%$ en mujeres. En cuanto al estado civil se encontró que el $43 \%$ estaba casado; 34\% viudo; $15 \%$ separado y el $8 \%$ soltero. Por su parte, el 33\% tiene una enfermedad crónica y el 75,3\% profesa una religión. En relación a las actividades del CAM (Centro del Adulto Mayor), el 71,3\% realiza una actividad fuera del centro del adulto mayor.

\section{Instrumentos.}

Escala de Resiliencia chilena (SV-RES). La validez y confiabilidad de la escala de resiliencia se realiza con el instrumento SV-RES elaborado por Saavedra y Villalta (2008b). Este instrumento cuenta con un formato de respuesta que va desde "muy de acuerdo" hasta "muy en desacuerdo", y contiene sesenta divididos. La prueba mide doce factores específicos de resiliencia: identidad, autonomía, satisfacción, pragmatismo, vínculos, redes, modelos, metas, afectividad, autoeficacia, aprendizaje y generatividad.

El instrumento fue aplicado a una muestra de 288 sujetos de ambos sexos entre las edades de quince y 65 años, pertenecientes a la comuna de Curicó. Arrojó una validez de 0,76 por medio de análisis test-retest y la confiabilidad medida a través del alpha de Cronbach es de 0,96. El objetivo del estudio era describir y comparar los puntajes generales y por factores de la variable de resiliencia. Asimismo, antes de su aplicación 
se realizó una prueba piloto en una muestra de 145 sujetos que arrojó una validez de 0,72 de confiabilidad.

Escala de Resiliencia Mexicana (RESI-M). Para la evidencia convergente se aplica la Escala de Resiliencia Mexicana (RESI-M). La construcción de esta escala se obtuvo a partir de dos instrumentos: la Escala de Resiliencia para Adultos (RSA) de Friborg y la versión de Connor y Davidson Resiliencie Scale (CD-RISC). Como resultado del análisis factorial emergieron cinco factores, todos ellos con alphas de Cronbach adecuados. El primer factor tuvo un alpha de 0,92, el segundo de 0,87, el tercero de 0,87, el cuarto de 0,84 y el último de 0,79. En total, el instrumento explica el $4361 \%$ de variancia y tiene una confiabilidad de 0,93. El RESI-M posee cinco factores: fortaleza y confianza en sí mismo, competencia social, apoyo familiar, apoyo social y estructura.

Procedimiento. En cuanto a la recolección de los datos, esta se realizó por medio de visitas a los centros del adulto mayor, en las cuales se aplicó un cuestionario sociodemográfico y posteriormente los instrumentos recolectores de la escala de resiliencia. A cada uno de los participantes se le brindó un consentimiento informado, en el que se detalla el objetivo de la investigación, carácter voluntario, la confidencialidad y anonimato de la recolección de datos. Asimismo, se solicitó autorización previa a la aplicación del instrumento recolector al gerente de desarrollo humano de la municipalidad mediante una carta de presentación gestionada a través de la Universidad de Ciencias Aplicada y la directora de adolescente de la municipalidad de Miraflores, con la que se hizo el primer contacto y nos refirió a los centros del adulto mayor.

Análisis de datos. En cuanto a la recolección de los datos, este se realizó por medio de visitas a los centros del adulto mayor, en las cuales se aplicó un cuestionario sociodemográfico y posteriormente los instrumentos recolectores de la Escala de Resiliencia. A cada uno de los participantes se les brindó un consentimiento informado, en donde se detalla el objetivo de la investigación, carácter voluntario, la confidencialidad y anonimato de la recolección de datos Asimismo, se solicitó autorización previa a la aplicación del instrumento recolector al gerente de desarrollo humano de la municipal mediante una carta de presentación gestionada a través de la Universidad de Ciencias Aplicada y la directora de adolescente de la municipalidad de Miraflores con la que se hizo el primer contacto y nos refirió a los centros del adulto mayor.

Los análisis de datos se elaboró mediante el programa SPSS Statistics realizando los análisis psicométricos de validez interna e externa. Como evidencia de validez en relación a la estructura interna se efectuó el análisis factorial exploratorio empleando el método de extracción de ejes principales (Frías y Pascual, 2012) frente a la posibilidad de que no se cumpla la normalidad multivariante con rotación oblicua, específicamente, Promax debido a que los factores correlacionan entre sí y es útil para conjuntos de datos grandes (De la fuente, 2011; citado en Frías y Pascual, 2012). Para la interpretación de las cargas factoriales se tomó en cuenta los valores de Stevens (1992; citado en Frías y Pascual, 2012) que propone que para que éstas sean aceptables deben ser mayores a .30. Para los análisis de correlación entre las variables, se examinó la distribución de los puntajes obtenidos mediante el análisis de normalidad para ambas escalas de Resiliencia. Para esto se aplicó la prueba Kolmogorov Smirnov ( $>50)$. Este test mostró que los puntajes de las variables psicológicas no se asemejan a la distribución normal, así los análisis fueron realizados utilizando la prueba de correlación de Spearman (correlación 
no paramétrica). Finalmente para los análisis de consistencia interna de utilizó la prueba de alfa de cronbach debido a que es el índice más utilizado para la evaluación de fiabilidad (Cicchetti, 1994; citado en Clark y Watson, 1995).

\section{Resultados}

A continuación, se presentarán los resultados de la investigación, en los cuales se describen las características psicométricas de los instrumentos utilizados. Primero se estudió la evidencia de validez mediante el análisis factorial exploratorio y luego se estudió la confiabilidad mediante la consistencia interna de la prueba (alpha de Cronbach).

Para recoger la evidencia de validez relacionada a la estructura interna de la escala de resiliencia SV-RES, se realizó un análisis factorial exploratorio mediante la factorización de ejes principales con rotación Promax. A partir de sus resultados se mostró que el test de Kaiser Meyer Olkin (KMO) de adecuación de la muestra alcanzó un valor de 0,946 y la prueba de esfericidad de Barlett fue significativa $\left(p<0,000 ; x^{2}=14.800,54 ; g l=1770\right)$. Los valores obtenidos indican que es adecuado efectuar un análisis factorial (Tabachnick y Fidell, 1989).

En los resultados iniciales se obtuvieron once componentes con autovalores mayores a uno que explicaban un 64,95\% de la varianza total. Debido a la dispersión de los ítems, no se pudo interpretar la solución factorial de los componentes y resulta de poca utilidad hacer un reporte de estos, ya que se encontraron dimensiones formadas con uno o dos ítems. Además, se observó el gráfico de sedimentación de Cattel, el cual indica la presencia de tres componentes. Estos hallazgos afirman la solución factorial encontrada en la base teórica de la prueba original (Grotberg, 2001).

Debido a ello, se realizó un análisis de ejes principales con ajuste a tres componentes. Al realizarse por segunda vez el análisis de componentes principales con rotación Promax, se obtuvieron componentes con autovalores mayores a uno que explicaban un 50,60\% de la varianza total. El primer factor obtuvo un autovalor de 10,97 que explicó el 18,29\% de la varianza que estuvo conformado por los ítems 1, 2, 3, 4, 5, 6, 7, 8, 9, 10,11, 12, $13,14,15,16,17,18,19,20,23$ y 24 que miden la categoría de "yo soy/yo estoy". El segundo factor obtuvo un autovalor de 8,46 que explicó el 14,11 de la varianza conformado por los ítems 21, 22, 27, 28, 29, 30, 31, 32, 33, 34, 35 y 36 pertenecientes a la categoría de "yo tengo". El tercer factor obtuvo un autovalor de 10,92 que explicó el $18,20 \%$ de la varianza que estuvo conformado por los ítems 37, 38, 39, 40, 41, 42, 43, $44,45,46,47,48,49,50,51,52,53,54,55,56,57,58,59$ y 60 que representan a la categoría de "yo puedo". Los ítems 25 y 26 obtuvieron cargas factoriales consideradas no aceptables ya que no superaron el valor mínimo recomendado de 0,30 (Stevens, 1992).

En la Tabla 1 se observaron cargas factoriales altas, pues superan el 0,60, las cuales son consideradas aceptables ya que superan el valor mínimo recomendado de 0,30 (Stevens, 1992). Las correlaciones totales de elementos corregida $(r)$ son adecuadas $(r>0,20)$ en todos los reactivos, lo cual indicó consistencia entre ellos y apropiados niveles de covariación con la estimación global (Merino-Soto, Navarro y García, 2014; citado en Domínguez y Merino, 2015). 
Tabla 1. Cargas factoriales y correlaciones elemento total corregida $(r)$ de la Escala SV-RES.

\begin{tabular}{lccccc}
\hline & Items & YS-E & YT & YP & $\boldsymbol{r}$ \\
\hline 8. Seguro de mí mismo. & & .82 & & .74
\end{tabular}

.78

68

9. Seguro de mis proyectos y metas.

14. Satisfecho con mis relaciones de amistad.

11. Una persona que ha aprendido a salir adelante en la vida.

17. Una persona con metas en la vida.

5. Creciendo como persona.

16. Una persona práctica.

7. En contacto con personas que me aprecian.

18. Activo frente a mis problemas.

4. Seguro de mis creencias o principios.

10. Seguro en el ambiente en que vivo.

6 . Rodeado de personas que en general me ayudan en situaciones

13. Bien integrado en mi lugar de trabajo o estudio.

12. Un modelo positivo para otras personas.

20. Generando soluciones a mis problemas

19. Revisando constantemente el sentido de mi vida

15. Satisfecho con mis relaciones afectivas.

3. Optimista respecto del futuro.

1. Una persona con esperanza.

.58

24. Fortaleza interior.

2. Una persona con buena autoestima.

.60

23. Relaciones afectivas sólidas.

.60

32. Personas que me han ayudado a evitar peligros o problemas

28. A quien recurrir en caso de problemas.

.70

33. Personas en las cuales puedo confiar.

.73

35. Personas que me han acompañado cuando he tenido problemas.

29. Personas que estimulan mi autoestima e iniciativa.

.69

31. Personas que me han orientado y aconsejado.

.60

38. Personas con quien enfrentar los problemas.

.72

34. Personas que han confiado sus problemas en mí.

.68

27. Personas que me apoyan.

.67

43. Confiar en las personas.

.61

21. Relaciones personales confiables.

39. Proyectos a futuro.

40. Problemas que puedo solucionar.

22. Una familia bien estructurada.

36. Metas a corto plazo.

30. Satisfacción con lo que he logrado en la vida.

47. Dar mi opinión.

53. Aprender de mis aciertos y errores.

52. Comunicarme adecuadamente.

.78

46. Resolver mis problemas de manera efectiva. 
57. Fijarme metas realistas.

55. Tomar decisiones.

50. Responsabilizarme por lo que hago.

49. Apoyar a otros que tienen dificultades.

45. Desarrollar vínculos afectivos.

56. Generar estrategias para solucionar problemas.

44. Superar las dificultades que se me presenten en la vida.

58. Esforzarme por lograr mis objetivos.

54. Colaborar con otros para mejorar la vida de mi comunidad.

60. Proyectarme al futuro.

48. Buscar ayuda cuando la necesito.

41. Hablar de mis emociones.

59. Asumir riesgos.

37. Mis objetivos claros.

Análisis de fiabilidad. En cuanto a la confiabilidad del factor "yo soy/yo estoy" a través del método de consistencia interna mediante el coeficiente alpha de Cronbach, se obtuvo como resultado un coeficiente de 0,95 [IC $95 \% ; 0,94,0, e 95$ ] y el rango de las correlaciones totales de elementos corregido osciló entre 0,58 y 0,76 . Asimismo, se calculó la confiabilidad del factor "yo puedo" en el que se obtuvo un coeficiente de 0,96 [IC $95 \% ; 0,94,0,96]$ y el rango de las correlaciones total de elementos corregido osciló entre 0,54 y 0,78 . Por último, se calculó la confiabilidad del factor "yo tengo" en el que se obtuvo un coeficiente de 0,94 [IC $95 \% ; 0,92,0,94$ ].

Evidencias de validez convergente. Se procedió a correlacionar los tres componentes de la solución final de la escala SV-RES con los cinco factores de la escala de RESIM. Para tal fin se utilizó la prueba de correlación de Spearman (correlación no paramétrica), ya que, en el análisis de normalidad, mediante la prueba de Kolmogorov-Smirnov, todas las distribuciones de las variables resultaron estadísticamente significativas $(p<0,5)$ por lo tanto, no se aproximaron a una distribución normal. Tal como se observa en la Tabla 2 , se encontraron correlaciones positivas, significativas y fuertes entre ambas escalas, por lo que se obtienen evidencias de validez convergente.

Tabla 2. Correlaciones entre la escala de resiliencia SV-RES y la escala de resiliencia RESI-M.

\begin{tabular}{lccccccc}
\hline & PS & AS & ES & RI & CM & YT & YP \\
\hline Apoyo social & $0,70^{* * *}$ & & & & & & \\
Estructura & $0,70^{* * *}$ & $0,81^{* * *}$ & & & & & \\
Relaciones interpersonales & $0,76^{* * *}$ & $0,73^{* * *}$ & $0,69^{* * *}$ & & & & \\
Confianza en sí mismo & $0,76^{* * *}$ & $0,68^{* *}$ & $0,66^{* *}$ & $0,79^{* * *}$ & & & \\
Yo tengo & $0,59^{* *}$ & $0,65^{* *}$ & $0,59^{* *}$ & $0,64^{* *}$ & $0,62^{* *}$ & & \\
Yo puedo & $0,63^{* *}$ & $0,64^{* *}$ & $0,61^{* *}$ & $0,67^{* *}$ & $0,63^{* *}$ & $0,81^{* * *}$ & \\
Yo soy/estoy & $0,59^{* *}$ & $0,56^{* *}$ & $0,57^{* *}$ & $0,60^{* *}$ & $0,61^{* *}$ & $0,77^{* * *}$ & $0,73^{* * *}$ \\
\hline
\end{tabular}

$*_{\mathrm{p}}<0,05 ; * * \mathrm{p}<0,01 ; * * * \mathrm{p}<0,001$ 


\section{Discusión}

En la presente investigación se tuvo como objetivo examinar las propiedades psicométricas de evidencia de validez y confiablidad de las puntuaciones obtenidas para la adaptación de la escala de Resiliencia SV-RES en una muestra de adultos mayores de 60 años de la ciudad de Lima, Perú. Los resultados presentaron que la escala de Resiliencia (SVRES) obtenida de la versión de Saavaedra y Villalta (2008b) evidenció una apropiada y satisfactoria validez de estructura interna, validez convergente, así como una adecuada confiabilidad de consistencia interna de la escala en los puntajes obtenidos. Lo cual confirma su uso para efectos de investigaciones futuras en muestras de adultos mayores peruanos.

En la evaluación de validez relacionada a la estructura interna del constructo al realizar el análisis factorial exploratorio de la prueba se pudo observar que el modelo de 12 factores de la prueba original no se mantuvo, sino que se cambió a una estructura de 3 sub-escalas. Ello difiere de la estructura original del cuestionario de Resilienca (SV-RES). Sin embargo, se encontró que la mejor solución factorial encontrada era consistente con la teoría base de las tres grandes subescalas: YO SOY/ESTOY, YO PUEDO Y YO TENGO propuesta por Grotberg (1995), por lo que el autor refiere que estos 12 factores de su prueba original se encuentran en dichas subescalas, los cuales fueron revisados y aprobados encontrándose validez de contenido (Saavadera y Villalta, 2008b). Así mismo, se obtuvo componentes con autovalores mayores a 1 que explican un $50.60 \%$ de la varianza con cargas factoriales altas. Estos datos ponen en manifiesto su contribución significativa a la medición de resiliencia y brindan una solidez factorial (Stevens, 1992).

Con respecto a la distribución de los ítems, en el análisis factorial exploratorio se puede observar ítems con conflictividad factorial debido a que existen ítems con cargas factoriales compartidas $(>.30)$ presentes en más de una dimensión, lo cual indica que ese item está siendo representado por más de un factor. Sobre esto Pérez y Medrano (2010) indican que se estaría reteniendo ítems complejos y soluciones factoriales insatisfactorias difíciles de interpretar. Así mismo, en los ítems "Una vida con sentido" y "Acceso a servicios Sociales-Públicos" presentan cargas factoriales menores a .30 lo cual indica una carga muy baja y no aceptable. Sin embargo, no se cuenta con investigaciones en donde se estudie la estructura interna de la prueba, por lo que cabe señalar que falta contar con investigaciones futuras para poder corroborar estas diferencias de distribución y dimensiones de la prueba original SV-RES.

En relación a la confiabilidad de los puntajes generados de la investigación dio como resultado un alfa $>.90$ para sus tres factores la cual indica la homogeneidad de los ítems del instrumento en relación con la característica que se pretende medir, se concluye que el instrumento en estudio tiene un coeficiente de confiabilidad muy alto, lo cual indica que es confiable e interpretable.

En la validez externa se encontraron correlaciones altas y muy significativas lo cual indica que se está midiendo adecuadamente el constructo. Dentro de los datos que sobresalieron, se encontró la correlación de Yo soy/Yo estoy con percepción y confianza en sí mismo. Estas correlaciones se pueden deber a que ambas están evaluando procesos de autoevaluación y manera de percibirse a sí mismo: segura, confiada, con esperanzas y expectativas hacia el futuro. Por lo tanto, estas dimensiones encuentran una relación 
entre ellas y evalúan procesos psíquicos como la autonomía, crecimiento personal y autoestima. Según Rodríguez (2008), dichos procesos dependen en gran medida en la manera más o menos airosa y exitosa, que cada individuo practica para lograr superar los conflictos y dificultades de la vida. Esta situación se encuentran presentes en la población adulto mayor, ya que, en esta etapa se activan dichos procesos (Resnick, 2014). Por lo tanto, el impulso y desarrollo de los procesos psicológicos mencionados anteriormente brinda, al adulto mayor, un bienestar psicológico debido que, a pesar de los cambios característicos presentes en esa edad tanto físicas como sociales y psicológicas logran adaptarse a ellos logrando el replanteamiento de nuevas metas y desafíos (Ortiz y Castro, 2009).

Así mismo, se encontró que la dimensión Yo puedo se correlacionó muy significativamente con relaciones interpersonales y estructura, esto se debe por la definición de dichas dimensiones, las cuales indican el desarrollo de las capacidades interpersonales y resolución de conflictos encontrados en la estructura del individuo conocido como las reglas y las actividades que les facilitan tener organización y orden en su vida (Friborg, 2001; citado en Palomar y Gómez, 2010). Lo cual se esperaría que mientras las personas desarrollen sus habilidades y estrategias de resolución de conflicto van a tener una mayor capacidad para relacionarse con los demás y poder enfrentarse adaptativamente a los problemas del entorno. De tal manera que la expresión de los pensamientos, sentimientos, control de comportamiento e impulsos son un medio de comunicación y relación eficaz con los demás. De acuerdo a esto, la adquisición de estos nuevos recursos puede reducir el impacto negativo de los eventos estresantes y una forma efectiva que facilitaría un envejecimiento satisfactorio (Taylor y Stanton, 2007).

Por último, se encontró que las variables de Yo tengo se correlacionó con el Apoyo Social, porque ambas dimensiones contribuyen al apoyo externo que el individuo pueda recibir de sus familiares, comunidad y amigos cercanos (Grotberg, 2003). En este sentido si es que la persona tiene los vínculos estables y las relaciones interpersonales dentro de su red social contribuye a una mejor apreciación subjetiva y funcional del individuo (Escobar, Puga y Martín, 2008). De tal manera que el adulto mayor sienta la ayuda de las personas de su entorno brindándole apoyo emocional conllevaría a la prevención de sentimientos de nostalgia, tristeza y soledad debido a la falta de apoyo social (Del Valle y Coll I, 2011; citado en Cerquera y Quintero 2015).

Finalmente, se puede decir que la SV-RES es un instrumento válido y confiable que puede ser utilizado en la población adulta mayor en el contexto peruano. El presente estudio es un punto de partida para otras investigaciones para indagar más sobre la estructura de la prueba para promover e incentivar los factores protectores de la resiliencia. A partir de lo encontrado en estas investigaciones se podría realizar estudios comparativos entre sectores sociodemográficos y género, en pacientes hospitalizados, con adultos mayores en asilados, entre otras.

Por último, se encontraron algunas limitaciones a los largo de esta investigación. En primer lugar, al momento de aplicar la prueba con grupos de más de 10 de participantes se tuvo que ir diciendo que la prueba era individual y no grupal. Por lo tanto como sugerencia se considera que la aplicación de la prueba debe de ser en grupo pequeños y en casos necesarios de manera casi individualizada. En segundo lugar, debido a que no se tiene estudios previos donde se haya trabajo los análisis psicométricos de la prueba, 
imposibilitó una discusión más amplia y comparativa de los resultados. En tercer lugar, a pesar de que la prueba es extensa se debe tomar en cuenta que la variable de resiliencia de por sí, es compleja, si bien el objetivo principal es la validación del instrumento no se puede dejar de lado que se investiga de forma paralela componentes emocionales y conductuales. De esta manera, el instrumento permite extraer información valiosa y relevante que aporte usos prácticos para futuras investigaciones tomando en cuenta el propósito por el cual se está aplicando dicha prueba psicométrica. Finalmente, debido a que es un estudio no probabilístico no se puede generalizar a la población, por tanto, se impulsa la aplicación de la prueba en estudios y muestras más amplios.

\section{Agradecimientos}

Agradezco a los autores por sus contribuciones en la adquisición de datos y diseños de la prueba original que fue base de este trabajo: Saavedra y Villalta (2008b); Palomar y Gómez (2010).

\section{Referencias}

Alcalde, A., Alcalde, M., y Palacios, A. (2011). Trauma resiliencia: niños con experiencia de vida en la calle. Athenea, pp. 47-79. http://hdl.handle.net/10757/620986

Becoña, E. (2006). Resiliencia: Definición, características y utilidad del concepto. Revista de psicología clínica, 11(3), 125-146. Recuperado de http://aepcp.net/ arc/01.2006(3).Becona.pdf

Benavides, H. (2012). Cambios demográficos y proyecciones de población. Lima, Perú: Instituto Nacional de Estadística e Informática. Recuperado de http://web2.ceplan. gob.pe/documents/10157/4c87bd0e-0538-4588-bc22-583ddaeb7514

Benavides, A. (2015). Gestión del riesgo del adulto mayor. En torno a la prevención, 14, 30-35. Recuperado de http://www.relaciger.org/revista/pdf/spa/doc1403/doc1403contenido.pdf

Benvenuto, F. (2015). Resiliencia en madres adolescentes institucionalizadas en dos programas de acogidas (Tesis de titulación). Universidad Peruana de Ciencias Aplicadas, Lima. http://hdl.handle.net/10757/621881

Cárdenas, A., y López, A. (2010). Resiliencia en la vejez. Revista Salud Pública, 13(3), 528-540. Recuperado de http://www.scielosp.org/pdf/rsap/v13n3/v13n3a14.pdf

Caycho-Rodriguez, T; Ventura-León, J; Garcia-Cadena,C y otros (2018). Evidencias psicométricas de una medida breve de resiliencia en adultos mayores peruanos no institucionalizados. Psychosocial Interventio, 27, 73-79. Scielo, 27(2) 73-79, 7. Recuperado de https://doi.org/10.5093/pi2018a6.

Cerquera, A. y Quintero, M. (2015). Reflexiones grupales en gerontologia: el envejecimiento normal y patológico. Revista Virtual de la Universidad Católica del Norte, 45 173-180. Recuperado de http://revistavirtual.ucn.edu.co/index.php/ RevistaUCN/article/view/665/1196 
Connor, K.M. y Davidson, J.R.T. (2003). Development of a new resilience scale: The Connor-Davidson resilience scale (CD-RISC). Depression and Anxiety, 18, 76-82.

Cortés, J., Flores, P., Gómez, C., y Reyes, K. (2012). Resiliencia y su relación con estilos de vida de los adultos mayores autovalentes. Ciencia y enfermería, 18(3), 73-81. Recuperado de: http://www.scielo.cl/pdf/cienf/v18n3/art 08.pdf

Clark, L; Watson, D. (1995). Constructing validity: Basis issues in objective scale development. Psychological assessment, 7, 309-319. Recuperado de http://dx.doi. org/10.1037/1040-3590.7.3.309

Domínguez, S. y Merino, C. (2015). ¿Por qué es importante reportan los intervalos de confianza del coeficiente alfa de Cronbach?. Revista Latinoamericana de Ciencias Sociales, Niñez y Juventud, 13 (2), 1326-1328.

Escobar,M. ; Puga, D. y Martin, M. (2008) Asociaciones entre a red social y la discapacidad al comienzo de la vejez en as ciudades de Madrid y Barcelona en 2005. Revista Española de Salud Pública. [online] 82 (6) 637-651. Recuperado en 18 de diciembre de 2018 de http:// scielo.iscii.es/scielo.php?script=sci arttext\&pid=S1135$\underline{5727200800600005 \& I n g=e s \& t \mid n g=e s}$

Fernández, A. (2004). La psicología de la vejez. Encuentros Multidisciplinares, 16. Recuperada de http://www.encuentros-multidisciplinares.org/Revistan\%C2\%BA16/ Roc\%C3\%ADo\%20Fernandez\%20Ballesteros.pdf

Frías-Navarro, D. y Pascual , M. (2012) PRÁCTICAS DEL ANÁLISIS FACTORIAL EXPLORATORIO (AFE) EN LA INVESTIGACIÓN SOBRE CONDUCTA DEL CONSUMIDOR Y MARKETING Suma Psicológica, 19 ( 1), 45-58

Gómez, B. (2010). Resiliencia individual y familiar. España: Asociación Vasco-Navarra. Recuperado de http://www.avntfvntf.com/imagenes/biblioteca/G\%C3\%B3mez, \%20 B. \%20Trab. \%203\%C2\%BA\%20BI\%2009-10.pdf

Grotberg, E (1995). A guide to promoting resilience in children: Strengthening the human spirit. The international resilience project, (8). Recuperado de https://bibalex.org/ baifa/Attachment/Documents/115519.pdf.

Grotberg, E. (2001). La resiliencia en el mundo de hoy. Barcelona, España: Gedisa.

Grotberg, E. H. (2003). Resilience for Today: Gaining Strength from Adversity. Greenwood, SC: Praeger Publishers.

Instituto Nacional de Estadística e Informática - INEI (2015). Perú: estimaciones y proyecciones de población 1950-2050. Lima, Perú: Instituto Nacional de Estadística e Informática.

Jaque, R., y Mardones, M. (2009). Perfil sociodemográfico de salud y funcionalidad en adultos mayores de la comuna de Chillán. Theoria, 18(2), 81-89. Recuperado de http://www.ubiobio.cl/miweb/webfile/media/194/v/v18-2/07.pdf 
Jiménez, M. (2008). "Resiliencia y vejez". Madrid, Portal Mayores, Informes Portal Mayores, no 80. Lecciones de Gerontología, XV. Recuperado de: http://www. imsersomayores.csic.es/documentos/documentos/jimenez-resiliencia-01.pdf.

Jiménez, M. (2011). La resiliencia, el tesoro de las personas mayores. Revista española de geriatría y gerontología, 46(2), 59-60. Recuperado de https://www.researchgate. net/publication/272157195 Resiliencia el tesoro de las personas mayores

Manciaux, M. (2005). La resiliencia: resistir y rehacerse. Madrid: Gedisa.

Naciones Unidas (2011). Seguimiento de la segunda asamblea mundial sobre el envejecimiento: informe del secretario general. En: Segunda Asamblea Mundial sobre el Envejecimiento. Nueva York, EEUU: ONU.

Orosco, C. (2015). Depresión y autoestima en adultos mayores institucionalizados y no institucionalizados en la ciudad de Lima. Persona, 18, 91-104. Recuperado de https://revistas.ulima.edu.pe/index.php/Persona/article/viewFile/500/474

Ortiz, J. y Castro, M. (2009) Bienestar Psicológico de los adultos mayores, su relación con la autoestima y la autoeficacia. Contribución de Enfermería. Ciencia y Enfermería. $15(1) ; 25-31$

Palacios, M. (1994). Envejecimiento exitoso en adultos mayores de 70 años del Centro Diurno de Cartago. Revista de trabajo social, 70, 55-31. Recuperado de http:// docplayer.es/43924706-Envejecimiento-exitoso-de-persona-adultos-mayores-de70-anos-del-centro-diurno-de-cartago.html

Palomar, J., y Gómez, N. (2010). Desarrollo de una escala de medición de la resiliencia con mexicanos (RESI-M). Interdisciplinaria Revista de psicología y ciencias afines, 27(1), 7-22. Recuperado de http://www.redalyc.org/articulo.oa?id=18014748002

Pérez, E.R., y Medrano, L. (2010) Análisis Factorial Exploratorio: Bases Conceptuales y Metodológicas. Revista Argentina de Ciencias del Comportamiento, 2(1) 58-66

Quiceno, J. y Vinaccia, S. (2011). Resiliencia: una perspectiva desde la enfermedad crónica en población adulta. Pensamiento Psicológico, 9(17), 69-82. Recuperado de http://www.redalyc.org/pdf/801/80122596006.pdf.

Quiroz, A. (2014). Calidad de vida en mujeres adulto mayor del grupo jóvenes por siempre (Tesis de titulación). Universidad Autónoma del Estado de México, México. Recuperado de http://ri.uaemex.mx/handle/20.500.11799/49225

Resnick, B. (2014). Resilience in older adults. Topics in Geriatric Rehabilitacion, 30, 155163. Recuperado de https://doi.org/10.1097/TGR.0000000000000024

Retiz, O. (2016). Resiliencia, bienestar y expresión artística en jóvenes en situación de pobreza. (Tesis de licenciatura, Pontificia Universidad Católica del Perú). Recuperado de file:///C://RETIZ FLORES OLENKA RESILENCIA.pdf 
Rodríguez, M. (2008). La percepción de la persona adulta mayor en la sociedad ramonense actual. Revista Pensamiento Actual. 8(10-11) 31-39

Saavedra, E., y Villalta, M. (2008a). Medición de las características resilientes, un estudio comparativo en personas entre 15 y 65 años. Liberabit, 14, 31-40.

Saavedra, E. y Villalta, M. (2008b). Escala de resiliencia SV-RES para jóvenes y adultos. Ceanim: Chile.

Saavedra, E. (2003). El Enfoque Cognitivo Procesal Sistémico, como posibilidad de Intervenir educativamente en la formación de sujetos Resilientes. España: Universidad de Valladolid Saavedra, E., Castro, A., y Inostroza, A. (2012). Niveles de resiliencia en adultos diagnosticados con y sin depresión. Pequén, 2, 161-184.

Salgado, A. (2005). Métodos e instrumentos para medir la resiliencia: Una alternativa peruana. Liberabit, 11, 41-48. Recuperado de http://www.revistaliberabit.com/es/ revistas/liberabit11/SALGADO.pdf

Stevens, J. (1992). Applied Multivariate Statistics for the Social Sciences (2a ed.). Hillsdale, New Jersey: Erlbau.

Severina, M. (2017). Calidad de vida y la capacidad resiliente de adultos mayores en situación de vulnerabilidad asistentes a la fundación ancianos de la paz. (Tesis de grado). Universidad mayor de San Ándres, Bolivia. Recuperado de https://repositorio. umsa.bo/handle/123456789/14872

Tabachnick, B. G., y Fidell, L. S. (1989). Using Multivariate Statistics. New York: Harper y Row, Publishers, Inc.

Taylor, S., y Stanton, A. (2007) Coping Resources, Coping Processes, and Mental Health . Annual Review of Clinical Psychology, 3, 129-153.

Wells, M. (2010). Resilience in older adults living in rural, suburban, and urban areas. Online Journal of Rural Nursing and Health Care, 10(2), 45-54. Recuperado de http://rnojournal.binghamton.edu/index.php/RNO/article/viewFile/55/46 